\title{
Eficácia do uso do laser de baixa potência para o tratamento da DTM: Revisão
}

\section{integrativa}

\author{
Effectiveness of using low level laser for the treatment of TMD: Integrative review \\ Eficacia en el uso del láser de baja potencia para el tratamiento de la DTM: Revisión integrativa
}

Camilla Amorim Acioli Borba

ORCID: https://orcid.org/0000-0001-8127-4000 Faculdade de Odontologia de Pernambuco, Brasil E-mail: camilla.borba@upe.br

Daniel José Silveira Tôrres

ORCID: https://orcid.org/0000-0002-9044-622X Faculdade de Odontologia de Pernambuco, Brasil E-mail:daniel.torres@upe.br

Esdras Gabriel Alves-Silva

ORCID: https://orcid.org/0000-0002-2309-1115

Universidade Estadual de Campinas, Brasil

E-mail: esdras0702@yahoo.com.br

Renata Araújo Gomes de Sá

ORCID: https://orcid.org/0000-0001-6550-8225

Faculdade de Odontologia de Pernambuco, Brasil E-mail: renata.sa@upe.br

Eloiza Leonardo de Melo

ORCID: https://orcid.org/0000-0001-5845-1478

Faculdade de Odontologia de Pernambuco, Brasil

E-mail:eloiza.leonardo@upe.br

Marleny Elizabeth Márquez de Martínez Gerbi

ORCID: https://orcid.org/0000-0001-9174-2541

Universidade de Pernambuco, Brazil

E-mail: marleny.gerbi@upe.br

Mávio Eduardo Azevedo Bispo

ORCID: https://orcid.org/0000-0002-3781-0272

Universidade de Pernambuco, Brasil

E-mail: meb@ecomp.upe.br

Maria Regina Almeida de Menezes

ORCID: https://orcid.org/0000-0003-3012-3979

Faculdade de Odontologia do Pernambuco, Brasil E-mail: regina.menezes@upe.br

\begin{abstract}
Resumo
As disfunções temporomandibulares são caracterizadas pela presença de dor, inchaço e disjunção da articulação temporomandibular e/ou da musculatura. Sendo assim, o uso do laser proporciona anestesia dessa região, diminuindo a inflamação e o desconforto. $\mathrm{O}$ objetivo deste trabalho foi realizar uma revisão integrativa da literatura sobre o uso do laser no tratamento da DTM, destacando benefícios, limitações, eficácia, relevância e impacto para saúde bucal. A pergunta norteadora é: Qual a eficácia do uso de laser de baixa potência no tratamento da DTM? Uma revisão integrativa da literatura foi realizada por meio de uma busca de artigos científicos nas bases de dados eletrônica Web of Science, PubMed e Medline, utilizando os descritores LLLT (low-level laser therapy), TMD (temporomandibular joint dysfunction) e Orofacial, indexados no período de 2015 a 2020 que tratavam de case reports, clinical trial, review, metaanalysis e randomized controlled e excluindos artigos de books and documents e editoriais, através da integração das palavras "AND" e "MORE". Após os critérios de elegibilidade foram analisados 7 artigos integralmente publicados em língua inglesa. Os estudos mostraram que o uso do laser auxilia no tratamento da DTM diminuindo o período de reparação da lesão, dor e inflamação devido a sua capacidade analgésica e anti-inflamatória. Sua eficácia se deve ao aumento de colágeno na ferida, estímulo à angiogênese e na proporção de células disponíveis para cicatrização. Contudo, se torna necessário que estudos continuem sendo realizados para que assuntos como este sejam disseminados para a população, já que essa técnica se mostra promissora.
\end{abstract}

Palavras-chave: Laser de baixa potência; DTM; Dor orofacial; ATM. 


\begin{abstract}
Temporomandibular disorders are characterized either by the presence of pain, swelling and disjunction of the temporomandibular joint or musculature. Thus, the use of the laser has great efficiency to anesthesia on this area, reducing inflammation and discomfort. The objective of this survey was to carry out an integrative review of the literature on the use of laser in the treatment of TMD, highlighting benefits, limitations, effectiveness, relevance and impact for oral health. The guiding question is: how effective is the use of low-level laser in the treatment of TMD? An integrative literature review had been done by searching scientific articles in the electronic databases Web of Science, PubMed and Medline, using the descriptors low-level laser therapy (LLLT), temporomandibular joint dysfunction (TMD) and orofacial, indexed in the period from 2015 to 2020 that dealt with case reports, clinical trial, review, metaanalysis and randomized controlled. There were excluded books and documents, and editorial articles, through the application of the words "and" and "more". After the eligibility criteria, 7 articles published in English were analyzed. Studies have shown that the use of laser helps in the treatment of TMD regarding the patient comfort, reducing the healing period of the injury, pain and inflammation due to its analgesic and anti-inflammatory capacity. Its effectiveness is due to the increase of collagen content in the wound, stimulating the angiogenesis and the proportion of cells available for healing. Nevertheless, it is required that studies continue to be carried out, so that, matters like these are spread around to population, since this technique is promising for the area.
\end{abstract}

Keywords: Low level laser; TMD; Orofacial pain; TMJ.

\title{
Resumen
}

Las disfunciones temporomandibulares son caracterizadas por la presencia de dolor, hinchazón y disyunción de la articulación temporomandibular y/o de la musculatura. De esta manera, el uso del láser tiene gran eficiencia en la anestesia de esta región, disminuyendo la inflamación y el incómodo. El objetivo de este trabajo fue realizar una revisión integrativa de la literatura actual sobre el uso del láser en el tratamiento de la DTM, destacando beneficios, limitaciones, eficacia, relevancia e impacto para la salud bucal y para la sociedad. La pregunta guía es: ¿Cuál es la eficacia del uso del láser de baja potencia en el tratamiento de la DTM? Una revisión integrativa de la literatura fue realizada por medio de una búsqueda de artículos científicos en las bases de datos electrónicas Web of Science, PubMed y Medline, utilizando los descriptores: "LLLT" (low-level laser therapy), "TMD" (temporomandibular joint dysfunction) y "Orofacial", indexados en el periodo de 2015 a 2020 que trataban de reportes de caso, ensayo clínico, revisión, metaanálisis y ensayo controlado aleatorio, y fueron excluídos artículos de libros, documentos y editoriales, por medio de la integración de las palabras "AND" y "MORE". Posteriormente a los criterios de elegibilidad fueron analizados 7 artículos integralmente publicados en lengua inglesa. Los estudios mostraron que el uso del láser asiste en el tratamiento de la DTM en lo que se refiere a la comodidad del paciente, disminuyendo el periodo de reparación de la lesión, dolor y inflamación debido a su capacidad analgésica y antiinflamatoria. Su eficacia se debe al aumento en el teor de colágeno de la ferida, estímulo a la angiogénesis y en la proporción de células disponibles para la cicatrización. Sin embargo, se vuelve necesario que los estudios continúen siendo realizados, para que asuntos como este sean diseminados para la población, ya que esta técnica se muestra prometedora.

Palabras clave: Láser de baja potencia; TMD; Dolor orofacial; ATM.

\section{Introdução}

Com o desenvolvimento do laser de baixa potência, vários progressos ocorreram no ramo da Odontologia para diagnóstico e tratamento. Essa técnica possui uma grande eficácia, independente do tratamento, mesmo que o seu mecanismo de ação ainda não seja claro para a comunidade acadêmica. (Zokaee et al, 2018).

A articulação temporomandibular é aquela que prende o crânio com a mandíbula, tendo grande importância para a expressão facial, assim como na locomoção e atividade dos músculos da mastigação. A utilização do laser de baixa potência apresenta grande melhora no tratamento da disfunção dessa articulação por estimular a regeneração tecidual. (Machado et al, 2016).

O termo desordens temporomandibulares (DTM) refere-se a uma série de doenças que envolvem as articulações temporomandibulares, músculos mastigatórios e estruturas relacionadas,, podendo também acometer indivíduos com outros distúrbios de sensibilidade à dor como a fibromialgia. (Lim et al, 2010). Entretanto, a etiopatogenia da DTM é incerta, porém a maioria dos pesquisadores converge para uma origem multifatorial, com causas biomecânicas, neuromusculares, biopsicossociais e biológicos. Por isso, o tratamento da DTM envolve uma aproximação pluridisciplinar, incluindo outros 
métodos de terapia, como a terapia manual, ultrassom, estimulação elétrica transcutânea (TENS) e a terapia a laser. (XU et al, 2018).

A fototerapia do laser tem sido usada para o tratamento de condições musculoesqueléticas dolorosas. Essa técnica consiste na aplicação da luz emitida na faixa de espectro de 600-1000 nm (vermelho visível a infravermelho), com irradiância de $5 \mathrm{~mW} / \mathrm{cm}^{2}$ a $5 \mathrm{~W} / \mathrm{cm}^{2}$ e com potência variando entre $1 \mathrm{~mW}$ até $10 \mathrm{~W}$, com feixes pulsados ou contínuos, e o tempo de tratamento de 30-60s por ponto (Zokaee et al, 2018). Assim, o laser de baixa potência é usado desde a década de 60, ganhando forças com o passar dos anos e apresentando-se atualmente como modalidade terapêutica adjuvante no tratamento da DTM, podendo ser somado a terapias alternativas para o aumento da eficácia. (Cetiner et al, 2006).

O mecanismo de ação do laser de baixa potência não é totalmente esclarecido, entretanto, algumas informações revelam o nível dos impactos do laser de baixa potência: o efeito anti-inflamatório deriva da capacidade comprovada dos lasers de limitar a liberação de mediadores inflamatórios, como bradicinina, histamina e, especialmente, prostaglandina durante a resposta inflamatória. Já o efeito analgésico do laser de baixa potência está relacionado à inibição de fibras nervosas, que retarda a velocidade da condução, reduz a amplitude dos potenciais de ação compostos e suprime a inflamação neurogênica. Ao estimular o metabolismo do colágeno e a proliferação de fibroblastos, o LLLT atua como um agente terapêutico na cicatrização de feridas. (Zokaee et al, 2018).

Dessa maneira, o objetivo do presente estudo é realizar uma revisão integrativa da literatura para investigar a eficácia do laser de baixa potência para o tratamento da DTM, já que esta é a condição mais comum de dor crônica orofacial, destacando seus benefícios, limitações, relevância e impacto para a saúde bucal e conforto dos pacientes.

\section{Metodologia}

Refere-se a uma revisão integrativa da literatura acadêmica, desenvolvida no ano de 2020, seguindo a metodologia de: reconhecimento do problema, pesquisa na literatura previamente publicada, avaliação dos dados, apresentação dos resultados e análise quantitativa com coleta de dados dos artigos previamente publicados, como mostra Pereira et al (2018). Além disso, houve a identificação das palavras-chaves, parâmetros de inclusão e exclusão de artigos, e verificação nas bases de dados. (Whittemore et al, 2005).

\subsection{Estratégia de Busca}

Nosso estudo teve como objetivo a análise do impacto do laser de baixa potência no tratamento da disfunção temporomandibular por meio de uma revisão integrativa, retratando no uso do laser desacompanhado, ou em junção com outras formas de terapia, almejando uma síntese de conhecimentos relacionados a esse assunto, incluindo os benefícios e eficiência, além do impacto na saúde bucal do paciente. Tal análise se torna relevante, pois poucos artigos foram encontrados, mas importantes para serem divididos com a sociedade e mundo acadêmico. Foram achados artigos fazendo análises da sua eficiência no tratamento da DTM e de outras condições de dor, relacionando o laser de baixa potência com terapias fonoaudiológicas assim, como o uso conjunto do laser com exercícios motores dos músculos da mastigação.

É válido ressaltar a utilização de três descritores: LLLT (low-level laser therapy), TMD (Temporomandibular Joint Dysfunction) e OROFACIAL. Esses descritores foram escolhidos com base no DECS, e com isso foram encontrados artigos que não se encaixam no objetivo da revisão, o que fez necessária uma filtragem manual para eliminar os artigos que tangenciam ou fugiam ao tema ou artigos incompletos.

Foram utilizadas as plataformas PubMed, MedLine e Web of Science, onde os descritores foram inseridos nos filtros de busca de cada plataforma, como mostra a figura 1 . 
Figura 1. Fluxograma de estratégia de busca nas bases de dados.

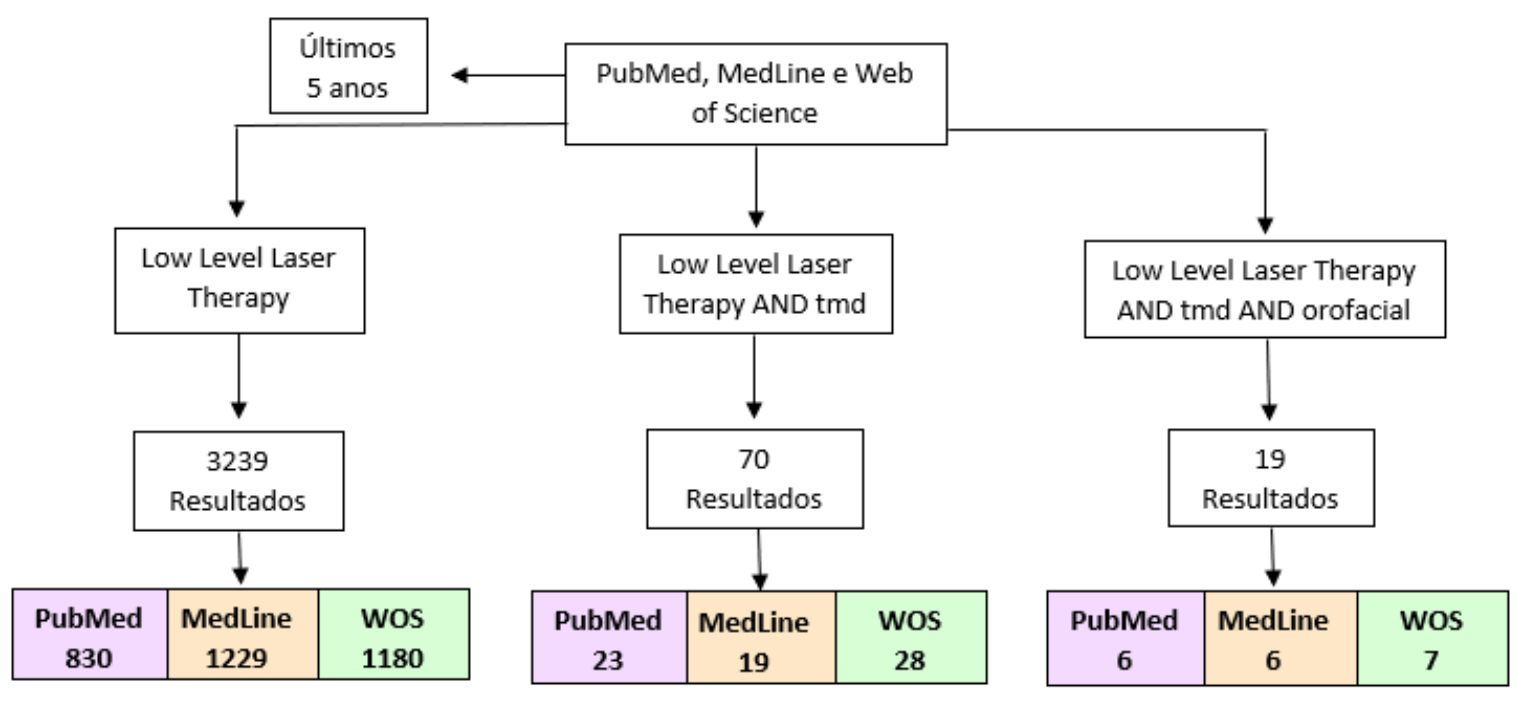

Fonte: Autores (2020)

A partir da busca de artigos científicos nas plataformas acima mencionadas no período de 2015 a outubro de 2020 utilizando o descritor "low level laser therapy (1llt)" obtivemos um total de 3239 artigos, após a junção desse descritor com “TMD” foi filtrado 70 resultados que já apareciam na pesquisa com apenas o primeiro descritor. Após a junção dos descritores anteriores mais "Orofacial" obtivemos uma amostra de 19 artigos também já apresentados nas duas outras pesquisas.

\subsection{Seleção das publicações e extração dos dados}

O processo de análise para avaliação e seleção dos artigos foi realizado por dois pesquisadores, de forma independente, com posterior confronto dos resultados para obtenção dos textos selecionados por consenso. Em casos de divergências ou dúvidas quanto à inclusão dos artigos, houve a participação de um terceiro pesquisador avaliador. Os artigos indexados repetidamente nos dois bancos de dados foram considerados apenas uma vez. Então, a seleção das publicações foi conduzida em duas fases: (1) leitura dos resumos, e (2) análise qualitativa dos textos na íntegra.

\subsection{Critérios de elegibilidade dos artigos}

Para seleção das fontes, foram consideradas como critérios de inclusão dos artigos originais disponibilizados na íntegra, publicados em língua inglesa e portuguesa indexados no períodos de 2015 a outubro de 2020 que tratavam de revisão de literatura, estudos in vitro, in vivo, relato de caso associando a laser de baixa potência e dor e/ou disjunção na articulação temporomandibular. Não foram incluídos textos incompletos, relato de caso de terapia com laser de baixa potência para outras disfunções, publicadas em outras línguas, ou em um período diferente do delimitado.

A Figura 2 ilustra a estratégia de busca de acordo com os critérios citados. 
Figura 2. Fluxograma de estratégia de busca e seleção dos artigos.

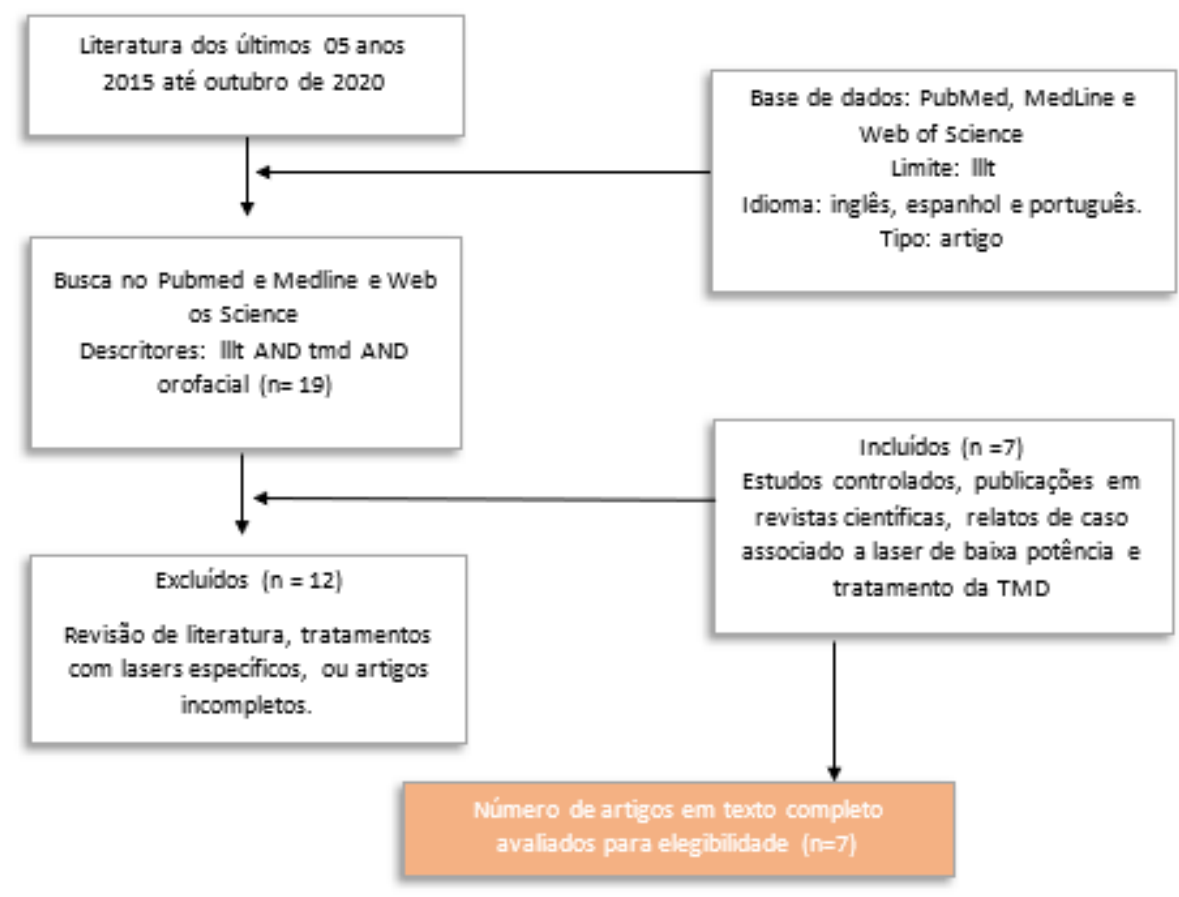

Fonte: Autores (2020)

Esta metodologia seguiu as recomendações PRISMA (Principais Itens para Relatar Revisões Sistemáticas e Metanálise). Os artigos que não se encaixavam nos critérios de inclusão, ou repetidos, foram excluídos. Posteriormente, dos artigos, restantes, foi realizada a leitura completa, chegando a uma amostra final de 7 artigos incluídos nesta revisão.

Não foram utilizadas teses, dissertações e monografias, visto que a realização de uma busca desses estudos é inviável logisticamente e a base de dados utilizada não tem esse tipo de publicação.

\section{Resultados}

Este estudo visou analisar a literatura acerca do uso de lasers de baixa potência, observando os benefícios, as limitações, as relevâncias e os impactos do uso na área odontológica. A presente revisão integrativa foi capaz de encontrar sete estudos recentes que demonstram a atuação do laser de baixa potência na odontologia. 
Quadro 1. Seleção dos artigos de acordo com o autor, ano, título e palavras-chave do artigo.

\begin{tabular}{|c|c|c|c|}
\hline Autor & Ano & Título & Palavras-Chave \\
\hline $\begin{array}{l}\text { De Oliveira, Dhelfeson } \\
\text { Willya Douglas et AL }\end{array}$ & 017 & $\begin{array}{l}\text { Do TMJ symptoms improve and last } \\
\text { across time after treatment with red } \\
(660 \mathrm{~nm}) \text { and infrared ( } 790 \mathrm{~nm}) \text { low } \\
\text { level laser treatment (LLLT)? A } \\
\text { survival analysis } \\
\text { Effects of oral motor exercises and } \\
\text { laser therapy on chronic } \\
\text { temporomandibular disorders: a } \\
\text { randomized study with follow-up }\end{array}$ & $\begin{array}{l}\text { - Laser } \\
\text { - Temporomandibular } \\
\text { - Survival analysis } \\
\text { - orofacial pain } \\
\text { - photobiomodulation } \\
\text { - Temporomandibular disorders } \\
\text { - Oral motor therapy } \\
\text { - Low-level laser therapy } \\
\text { - Rehabilitation } \\
\text { - Orofacial functions }\end{array}$ \\
\hline Zokaee, Haleh et al. & 018 & $\begin{array}{l}\text { Efficacy of Low-Level Laser } \\
\text { Therapy on Orofacial Pain: } \\
\text { A Literature Review }\end{array}$ & $\begin{array}{l}\text { - Headache } \\
\text { - low-level laser therapy } \\
\text { - mucositis } \\
\text { - temporomandibular disease }\end{array}$ \\
\hline Melchior et al. & 016 & $\begin{array}{l}\text { Efeito do tratamento } \\
\text { fonoaudiológico após a laserterapia } \\
\text { de baixa intensidade em pacientes } \\
\text { com DTM: estudo descritivo }\end{array}$ & $\begin{array}{l}\text {-Temporomandibular Joint Disorders } \\
\text { - Myofunctional Therapy } \\
\text { - Low Level Laser Therapy }\end{array}$ \\
\hline Panhoca, Vitor Hugo et al. & 015 & $\begin{array}{l}\text { Comparative clinical study of light } \\
\text { analgesic effect on } \\
\text { temporomandibular disorder (TMD) } \\
\text { using red and infrared led therapy }\end{array}$ & $\begin{array}{l}\text { - Laser therapy } \\
\text { - LED therapy } \\
\text { - TMD } \\
\text { - Pain relief } \\
\text { - Oral aperture }\end{array}$ \\
\hline $\begin{array}{l}\text { Rodrigues, } \quad \text { Carolina } \\
\text { Almeida et al. }\end{array}$ & 018 & $\begin{array}{l}\text { Can the severity of orofacial } \\
\text { myofunctional conditions interfere } \\
\text { with the response of analgesia } \\
\text { promoted by active or placebo low- } \\
\text { level laser therapy? }\end{array}$ & $\begin{array}{l}\text { - Laser therapy } \\
\text { - LED therapy } \\
\text { - TMD } \\
\text { - Pain relief } \\
\text { - Oral aperture }\end{array}$ \\
\hline $\begin{array}{l}\text { Magri, Laís Valencise et } \\
\text { al. }\end{array}$ & 017 & $\begin{array}{l}\text { Effectiveness of low-level laser } \\
\text { therapy on pain intensity, pressure } \\
\text { pain threshold, and SF-MPQ } \\
\text { indexes of women with myofascial } \\
\text { pain }\end{array}$ & $\begin{array}{l}\text { - Myofasial pain syndromes } \\
\text { - Temporomandibular joint disorders } \\
\text { - low-level laser therapy } \\
\text { - Pain measurement }\end{array}$ \\
\hline
\end{tabular}

Fonte: Autores (2020)

Foram selecionados, dentre os critérios, 7 artigos científicos. Os resultados revelam o uso do laser de baixa potência como técnica eficiente para o tratamento de Disfunção Temporomandibular, especialmente quando utilizado em conjunto com outras formas de terapia. Este estudo mostrou-se bem sucedido, embora requeira um maior número de pesquisas clínicas, com um grupo amostral mais vago e mais técnicas conjuntas para se obter conclusões mais expressivas. 


\section{Discussão}

O tratamento para DTM, como mostra Machado et al (2016), mesmo com o uso de terapias convencionais, como, por exemplo, o uso de placas interoclusais, ainda é um assunto controverso e debatido. A aplicação do laser de baixa potência para o tratamento da DTM apresenta benefícios que vão desde a estimulação de reações químicas que causam efeito anti-inflamatório e analgésico nas regiões afetadas, até atividade antimicrobiana, melhoria da abertura máxima e eliminação de sons em DTMs. Acredita-se que o efeito anti-inflamatório dos lasers de baixo nível deriva da capacidade comprovada dos lasers de limitar a liberação de substâncias responsáveis por mediar os processos inflamatórios, como bradicinina, histamina e especialmente prostaglandina durante as respostas inflamatórias, e seu efeito analgésico está relacionado à inibição das fibras A $\delta$ e $\mathrm{C}$, que retardam a velocidade de condução, reduzem a amplitude dos potenciais de ação compostos e suprimem a inflamação neurogênica. Além disso, a mucosite, inflamação nas membranas mucosas, pode ser prevenida ou tratada por aplicações profiláticas ou uso empírico. (Zokaee et al, 2018).

Quanto à eficácia do tratamento, Zokaee et al (2018) revela que a ação do laser de baixa potência gera uma atividade antimicrobiana, que contribui para a aceleração da cicatrização de feridas e a deposição óssea. Além disso, há uma redução significativa da dor através da modulação do processo inflamatório, que contribui para a melhoria do movimento da mandíbula, da dificuldade na mastigação e do desempenho mastigatório.

A utilização do laser, aliado a outros métodos de tratamento, garante uma resposta ainda mais eficiente. Como apresenta Melchior et al (2016), o tratamento com laserterapia seguido da terapia fonoaudiológica miofuncional orofacial, acarreta a diminuição dos sinais e sintomas remanescentes da DTM. Além disso, Machado et al (2016) mostra que a combinação do laser de baixa potência com exercícios orofaciais também tem a capacidade de reduzir os sintomas, entretanto, não substitui a terapia miofuncional orofacial completa.

Quanto à limitação de seu uso, não se sabe o porquê de, em alguns casos isolados, não haver diferença significativa na melhoria da dor em voluntários submetidos à terapia com laser, em relação àqueles do grupo placebo. Porém, Zokaee et al (2018) e Machado et al (2016) mostram que o uso do laser ativo e como placebo ajuda a diminuir a dor ligadas aos músculos da mastigação e melhora o estágio de recuperação após procedimentos orofaciais, revelando a necessidade de estudos avançados e com maior espaço amostral para uma conclusão, mesmo que o uso do laser seja, até agora, promissor.

Uma vez que 40 a 75\% da população apresentam algum sinal de DTM e 33\% apresentam algum sintoma, sendo a dor o mais frequente, principalmente nos músculos da mastigação e na região pré-auricular, assim, essa condição musculoesquelética se torna a maior causa de dor não dental na região orofacial. Dessa forma, é possível avaliar o impacto do laser de baixa potência no tratamento da DTM para promover a saúde bucal dessa parcela significativa da população (MAIA et al, 2012). Embora possa ocorrer em qualquer indivíduo, há uma prevalência maior nas mulheres e, como mostra MAGRI et al, (2017), mulheres com DTM frequentemente apresentam dor em outras áreas do corpo causadas pelo fenômeno da sensibilização central, a qual também foi reduzida pelo tratamento com laser de baixa potência.

Nesse contexto, nosso estudo buscou, por meio de uma revisão integrativa, analisar estudos relevantes sobre a aplicação do laser de baixa potência, com objetivo de aprimorar o tratamento da disfunção temporomandibular, fazendo uma síntese dos conhecimentos relacionados a esse assunto, incluindo os benefícios e limitações, além do impacto na saúde bucal. Tal estudo se torna relevante, pois encontramos poucos estudos nessa área, mas estes foram unânimes ao relatar a eficácia do laser de baixa potência no tratamento da DTM, o que os torna importantes de serem compartilhados com a sociedade e com o universo acadêmico

Importante pontuar que, ao colocarmos os descritores "Low level laser therapy", "TMD” e "orofacial”, escolhidos a partir de uma análise no DECS, foram encontrados trabalhos que não eram compatíveis com o objetivo da revisão integrativa, o 
que tornou necessária uma filtragem manual para a eliminação de artigos que tangenciavam o tema escolhido e artigos que não possuíam informações básicas que agregassem à revisão integrativa.

No PubMed, ao utilizarmos os três descritores em seu banco de dados, foram encontrados seis artigos. Contudo, um artigo foi excluído por tangenciar o tema escolhido e, dos cinco escolhidos, quatro estavam presentes nas outras plataformas e o último também estava presente no Web of Science.

Já no MedLine, usando os três descritores, foram encontrados seis artigos. Entre eles, um foi excluído por tangenciar o tema, quatro dos restantes por já terem sido escolhidos no PubMed, restando apenas um pertinente ao tema exposto e com todas as informações básicas.

Por sua vez, no banco de dados do Web of Science, usando os três descritores, foram encontrados sete artigos válidos ao tema, sendo seis deles já escolhidos em outras plataformas, ficando apenas um, que também foi incluído por conter as informações básicas para preenchimento da tabela e ser relevante para o estudo.

Foi importante o uso do conectivo AND para afunilar e obtermos tais resultados, vale salientar que apenas os artigos completos foram incluídos, pois muitos, mesmo possuindo resumo, não ofereciam dados inteiros capazes de enriquecer a pesquisa. Tornou-se relevante que os artigos descrevessem, com objetividade e clareza, como foi realizada a busca e uso das plataformas. Tais sites de buscas, PubMed, MedLine e Web of Science são referências mundiais, o que ajudou na pesquisa realizada.

\section{Considerações Finais}

É evidente que a literatura converge quanto ao uso do laser de baixa potência, ao afirmar sua eficiência e praticidade ao tratar as dores e reduzir o desconforto dos pacientes com Disfunção Temporomandibular. Com seu efeito analgésico e antiinflamatório, a laserterapia possibilita uma rápida recuperação da lesão e reduz a probabilidade de problemas derivados da contusão.

É importante ressaltar a grande quantidade de pessoas acometidas com as disfunções temporomandibulares, já que 33\% da população adulta já apresentou algum sinal ou sintoma, tendo 15,6\% desses a necessidade de tratamento, como mostra o Protocolo de Disfunção Temporomandibular e Dor Orofacial da Red SUSBH.

Todavia, é nítida a necessidade de mais análises e padronização da técnica, pois há diversos protocolos que divergem entre si, além da necessidade da produção de novos artigos no cenário acadêmico, divulgando novos métodos e diferentes pontos de vista, e, desse modo, é evidenciada, novamente, a relevância desta revisão integrativa, pois pode servir de análise para novas pesquisas.

Para trabalhos futuros, que utilizem desta revisão integrativa como base, é recomendada uma maior pesquisa, com descritores mais amplos, para que artigos que não foram incluídos e que talvez pertinentes, possam ser estudados e analisados pelos pesquisadores.

\section{Referências}

Cetiner, S., Kahraman, S. A., \& Yücetaş, S. (2006). Evaluation of low-level laser therapy in the treatment of temporomandibular disorders. Photomedicine and laser surgery, 24(5), 637-641. https://doi.org/10.1089/pho.2006.24.637.

Carrasco, T. G., Mazzetto, M. O., Mazzetto, R. G., \& Mestriner, W., Jr (2008). Low intensity laser therapy in temporomandibular disorder: a phase II doubleblind study. Cranio : the journal of craniomandibular practice, 26(4), 274-281. https://doi.org/10.1179/crn.2008.037

Douglas De Oliveira, D. W., Lages, F. S., Guimarães, R. C., Pereira, T. S., Botelho, A. M., Glória, J., Tavano, K., Gonçalves, P. F., \& Flecha, O. D. (2017). Do TMJ symptoms improve and last across time after treatment with red (660 nm) and infrared (790 nm) low level laser treatment (LLLT)? A survival analysis. Cranio : the journal of craniomandibular practice, 35(6), 372-378. https://doi.org/10.1080/08869634.2017. 
Emshoff, R., Bösch, R., Pümpel, E., Schöning, H., \& Strobl, H. (2008). Low-level laser therapy for treatment of temporomandibular joint pain: a double-blind and placebo-controlled trial. Oral surgery, oral medicine, oral pathology, oral radiology, and endodontics, 105(4), 452-456. https://doi.org/10.1016/j.tripleo.2007.09.012

Fernandes, T. de F., Pitchon, A. \& Pedras, R. B. (2016). Protocolo de disfunção temporomandibular/dtm e dor orofacial/dorf da rede SUSBH.

Lim, P. F., Smith, S., Bhalang, K., Slade, G. D., \& Maixner, W. (2010). Development of temporomandibular disorders is associated with greater bodily pain experience. The Clinical journal of pain, 26(2), 116-120. https://doi.org/10.1097/AJP.0b013e3181c507ef.

Machado, B. C., Mazzetto, M. O., Da Silva, M. A., \& de Felício, C. M. (2016). Effects of oral motor exercises and laser therapy on chronic temporomandibular disorders: a randomized study with follow-up. Lasers in medical science, 31(5), 945-954. https://doi.org/10.1007/s10103-016-1935-6.

Magri, L. V., Carvalho, V. A., Rodrigues, F. C., Bataglion, C., \& Leite-Panissi, C. R. (2017). Effectiveness of low-level laser therapy on pain intensity, pressure pain threshold, and SF-MPQ indexes of women with myofascial pain. Lasers in medical science, 32(2), 419-428. https://doi.org/10.1007/s10103-016-2138-x.

Maia, M. L., Bonjardim, L. R., Quintans, J., Ribeiro, M. A., Maia, L. G., \& Conti, P. C. (2012). Effect of low-level laser therapy on pain levels in patients with temporomandibular disorders: a systematic review. Journal of applied oral science: revista FOB, 20(6), 594-602. https://doi.org/10.1590/s167877572012000600002

Melchior, M. de O., et al. (2016). Efeito do tratamento fonoaudiológico após a laserterapia de baixa intensidade em pacientes com DTM: estudo descritivo. CoDAS, 28(6), 818-822. https://doi.org/10.1590/2317-1782/20162015099.

Panhoca, V. H., Lizarelli, R., Nunez, S. C., Pizzo, R. C., Grecco, C., Paolillo, F. R., \& Bagnato, V. S. (2015). Comparative clinical study of light analgesic effect on temporomandibular disorder (TMD) using red and infrared led therapy. Lasers in medical science, 30(2), 815-822. https://doi.org/10.1007/s10103-0131444-9.

Pereira, A. S., Shitsuka, D. M., Parreira, F. J., \& Shitsuka, R. (2018). Metodologia da pesquisa científica.

Petrucci, A., Sgolastra, F., Gatto, R., Mattei, A., \& Monaco, A. (2011). Effectiveness of low-level laser therapy in temporomandibular disorders: a systematic review and meta-analysis. Journal of orofacial pain, 25(4), 298-307.

Rodrigues, C. A., Melchior, M. O., Valencise Magri, L., \& Mazzetto, M. O.(2020). Can the severity of orofacial myofunctional conditions interfere with the response of analgesia promoted by active or placebo low-level laser therapy? Cranio: the journal of craniomandibular practice, 38(4), 240-247. https://doi.org/10.1080/08869634.2018.1520950

Venancio, R., Camparis, C. M., \& Lizarelli, R. (2005). Low intensity laser therapy in the treatment of temporomandibular disorders: a double-blind study. Journal of oral rehabilitation, 32(11), 800-807. https://doi.org/10.1111/j.1365-2842.2005.01516.x

Whittemore, R., \& Knafl, K. (2005). The integrative review: updated methodology. Journal of advanced nursing, 52(5), 546-553. https://doi.org/10.1111/j.13652648.2005.03621.x.

Xu, G. Z., Jia, J., Jin, L., Li, J. H., Wang, Z. Y., \& Cao, D. Y. (2018). Low-Level Laser Therapy for Temporomandibular Disorders: A Systematic Review with Meta-Analysis. Pain research \& management, 2018, 4230583. https://doi.org/10.1155/2018/4230583

Zokaee, H., Zahmati, A. H. A., Mojrian, N., Boostani, A., \& Vaghari, M. (2018). Efficacy of Low Level Laser Therapy on Orofacial Pain: A Literature Review. Advances in human biology, 8(2), 70-73. https://doi.org/10.4103/AIHB.AIHB_2_18. 